\title{
Integrating science and clinical practice in the understanding and treatment of mood disorders
}

\author{
Hagop Akiskal \\ From $1^{\text {st }}$ International Congress on Neurobiology and Clinical Psychopharmacology and European \\ Psychiatric Association Conference on Treatment Guidance \\ Thessaloniki, Greece. 19-22 November 2009
}

The Hippocratic humoral theory is upheld in modern neuropharmacologic investigations showing depressive relapse with catecholamine-depleting agents. Serotonin dysregulation is supported, especially in females, in reduced 5-HT2A receptors in PET studies. Furthermore, the ancient concept of temperament and its role in depression is supported by current psychometric research. Despite increasing clinical and public health recognition, sophisticated research strategies and the broad availability of relatively safer agents for treatment, depression continues to be a prevalent group of illnesses which often pursue a recurrent and chronically relapsing course with considerable morbidity and mortality. Etiology is multifactorial, involving, among others, familial-genetic, demographic, developmental, personality, seasonal-circadian, experiential, interpersonal, conjugal, cultural and economic factors. Grief, losses and other life events, emphasized in Freud's and Bowlby's writings, as well as current clinical research experience, together with Darwin's landmark work on evolution, place sadness and related emotions in an adaptive context. Current evidence indicates that experiencing life events depends on a familial diathesis for depression, and that temperament increases reactivity to the very life events that precipitate depression. Even social support, a factor that buffers the depressant effect of life events, appears to be dependent on familial factors. Finally, a positive family history of depression, seems to involve the amygdala. It is of great theoretical and clinical interest that the responsivity of the amygdala is mediated through such conditions as anxiety, bipolar II and related affective

International Mood Center at the University of California at San Diego, USA personality styles. Response of aversive stimuli, too, appears to be processed through the limbic structures. Many medically associated somatic complaints-fibromyalgia, irritable bowel syndrome and migraineappear to be part of the foregoing affective spectrum. Depression may precede, follow or complicate such common diseases as diabetes, myocardial infarction, stroke, and treatment of the associated depression often improves the prognosis of the underlying disease. Another provocative development is the continuity of unipolar and bipolarI II doisorders, which may in selected patients necessitate the use of antidepressant augmentation strategies.

Current treatment research involves complex methodologies in both the psychosocial and psychopharmacologic realms, which each clinician must practice as an art. Medications are best administered in the context of of an ongoing therapeutic alliance. Prototypes of modern mood stabilizers [lithium, carbamazepine, divalproex, topiramate, gabapentin, lamotrigene] are efficaceous via somewhat overlapping yet distinct mechanisms. Many antipsychotics [eg, perphenazine, risperidone, olanzapine, quetiapine, ziprasidone, aripiprazole] exercise prominent mood stabilizing effects. Finally, prototypes of antidepressants exert thymoleptic action via putative noradrenergic [desipramine], serotoninergic [fluoxetine], or dual chemical action[ venlafaxine], possibly dopaminergic [bupropion?], and in the case of MAOIs such as tranylcypromine appear to involve all three neurotransmitters. Glutaminergic action represents a new fascinating vista beyond the scope of this presentation. Given that mood disorders do occur in a distinct minority of eminent and creative individuals, the clinician can appropriately weigh the 
relative advantages and limitations of these agents as well as their different side-effect profiles for a given patient in these populations.

Published: 22 April 2010

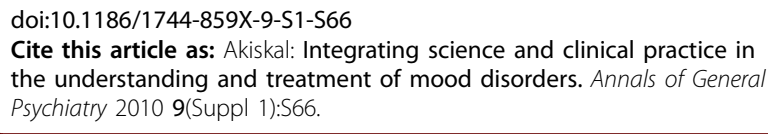

Submit your next manuscript to BioMed Central and take full advantage of:

- Convenient online submission

- Thorough peer review

- No space constraints or color figure charges

- Immediate publication on acceptance

- Inclusion in PubMed, CAS, Scopus and Google Scholar

- Research which is freely available for redistribution 